INRA Prod. Anim.,

2013, $26(5), 415-424$

\title{
| La fonte lipidique du foie gras à la cuisson
}

\author{
L. THÉRON ${ }^{1,2,3}$, M. BOUILLIER-OUDOT T, ${ }^{2,1,3}$, C. MARIE-ETANCELIN ${ }^{6}$, \\ C. BONNEFONT ${ }^{2,1,3}$, X. FERNANDEZ $Z^{1,2,3}$, C. MOLETTE ${ }^{2,1,3,4,5}$ \\ I INRA, UMR1289 TANDEM, F-31326 Castanet-Tolosan, France \\ 2 Université de Toulouse, INPT ENSAT, UMR1289 TANDEM, F-31326 Castanet-Tolosan, France \\ ${ }^{3}$ Université de Toulouse, INPT ENVT, UMR1289 TANDEM, F-31076 Toulouse, France \\ ${ }^{4}$ INRA, UMR444 Génétique Cellulaire, F-31326 Castanet-Tolosan, France \\ ${ }_{5}^{5}$ Université de Toulouse, INPT ENVT, UMR444 Génétique Cellulaire, F-31076 Toulouse, France \\ ${ }^{6}$ INRA, UR0631 SAGA, F-31326 Castanet-Tolosan, France \\ Courriel:molette@ensat.fr
}

La connaissance des facteurs biologiques, zootechniques et technologiques ainsi que des mécanismes qui contrôlent le taux de fonte des foies gras à la cuisson est primordiale pour la maîtrise du rendement technologique des foies gras. En effet, le taux de fonte à la cuisson a des répercussions sur les qualités organoleptiques des foies gras et sur les performances des unités de production.

La capacité à retenir les lipides lors de la cuisson constitue la principale qualité technologique recherchée du foie gras. La fonte se traduit par une exsudation de nature essentiellement lipidique (les lipides représentent de 90 à $100 \%$ du poids sec de l'exsudat chez le canard; Fernandez, résultats non publiés) qui a lieu lors de tout traitement thermique de conservation : pasteurisation ou stérilisation, par exemple. Le taux de fonte lipidique est calculé comme le rapport entre les exsudats et le poids de foie frais et il est exprimé en pourcentage. Le rendement technologique se déduit du taux de fonte par la formule suivante : 100 - taux de fonte, il est aussi exprimé en pourcentage.

La fonte lipidique à la cuisson du foie gras constitue une préoccupation pour les acteurs de la filière « foie gras ». En effet, le taux de fonte lipidique à la cuisson est soumis à réglementation. Le décret $n^{\circ}$ 93-999 du 9 août 1993, relatif aux préparations à base de foie gras, stipule que les produits à dénomination « foie gras » ne doivent pas comporter plus de $30 \%$ de graisse exsudée. Parmi les produits issus du gavage des palmipèdes, les produits à dénomination « foie gras entier » ont la meilleure valeur ajoutée, deux fois plus importante que celle du foie gras en bloc (Comité Interprofessionnel du Foie Gras, 2011). De l'aptitude à la transformation des foies gras dépend donc la rentabilité de la chaîne de production.

De plus, la variabilité de la fonte lipidique à la cuisson induit une hétérogénéité des produits finis, qui porte sur le poids net de foie consommable, la présentation du produit et ses qualités organoleptiques. Dans un contexte industriel, l'uniformité du produit vendu est primordiale tant pour le transformateur que pour le consommateur. Si cette variabilité du taux de fonte reste limitée pour des gammes de poids de foies gras faibles, elle augmente pour des gammes de poids plus élevées.

Ainsi, face à cette préoccupation récurrente, aujourd'hui, le poids de foie est la seule clé de tri objective disponible pour les transformateurs permettant de limiter la variabilité de la fonte à la cuisson. En industrie, un tri sur des paramètres de texture appréciés manuellement par des personnes qualifiées et entraînées est également effectué. Mais dans la mesure où ce tri est dépendant de l'opérateur, cela demeure une clé de tri subjective.

Il est donc primordial pour l'ensemble de la filière " foie gras » de mesurer, comprendre et maîtriser la fonte lipidique à la cuisson.

\section{1 / Méthodes d'évaluation de la fonte à la cuisson}

Des méthodes de mesure de la fonte à la cuisson ont été développées en laboratoire pour évaluer le rendement technologique des produits finis. Ce dernier est alors utilisé comme un facteur de comparaison dans les études sur le déterminisme de la fonte. D'autres méthodes ont été développées pour, ou par les transformateurs comme moyen de prédiction afin d'orienter la matière première vers la chaîne de transformation la plus adaptée. Les foies ayant de bons rendements prédits seront préparés en foie gras entier alors que des foies ayant des rendements prédits faibles subiront une émulsion pour constituer des blocs de foie gras.

Les principales méthodes d'évaluation de la fonte à la cuisson sont la mesure en verrine ou en boîte, le test « pilulier» et le « qualimètre ».

La mesure en verrine (180 g) est effectuée après pasteurisation du foie gras dans un autoclave à eau. Après ouverture des verrines dans une pièce climatisée à $10^{\circ} \mathrm{C}$, la graisse exsudée en périphérie du produit est retirée manuellement par grattage et le rendement technologique est déterminé par le rapport du poids de foie cuit sans la graisse exsudée sur le poids de foie avant cuisson. Même s'il est difficile d'obtenir des informations sur les barèmes exacts de température employés dans l'industrie, le mode de cuisson utilisé (pasteurisation vs stérilisation) semble seulement influencer la valeur absolue du rendement technologique (figure 1). Aussi, bien qu'il s'agisse d'une méthode exclusivement réservée à l'expérimentation (UMR TANDEM) de par son caractère destructif, elle constitue un très bon indicateur de la hiérarchie de fonte des foies gras entiers transformés en verrine en milieu industriel.

Le test en "boîte " est aussi un test couramment réalisé dans un contexte expérimental (Unité Expérimentale INRA Palmipèdes à Foie Gras). Un échantillon 
Figure 1. Relation entre le rendement technologique suite à une pasteurisation (Valeur pasteurisatrice, VP $=200$ ) et une stérilisation (Valeur stérilisatrice, VS = $0,75)$ effectuées sur les mêmes foies gras de canard mulard $(n=97)$.

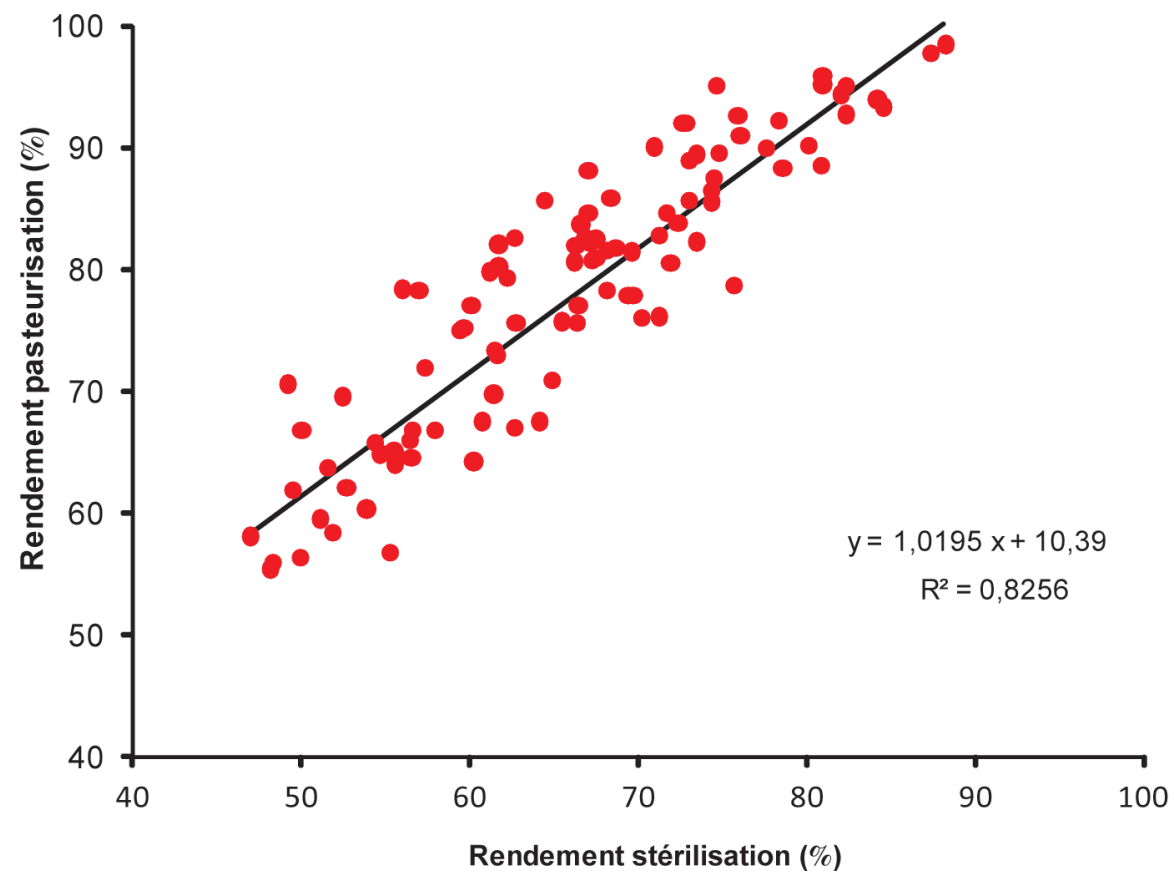

(60 g) est prélevé, placé dans une boîte en fer qui est sertie. Les boîtes sont placées dans l'autoclave pendant $65 \mathrm{~min}$ à $105^{\circ} \mathrm{C}$ (Valeur Stérilisatrice, VS $=0,8$ ). Après quelques jours de stockage en chambre froide, les boîtes sont placées au bain-marie pendant $10 \mathrm{~min}$ à $70^{\circ} \mathrm{C}$ afin que 1'exsudat lipidique devienne liquide et puisse être écarté facilement à l'ouverture de la boîte. Le foie restant est pesé et le rendement s'exprime de la même manière que précédemment décrit. Cette méthode, destructive comme la précédente, permet d'obtenir une indication précise de la fonte totale subie par le foie.

Le test en "pilulier » est pratiqué par certaines entreprises de transformation de foies gras entiers. Il s'agit d'un des critères de sélection pour la transformation. Une petite portion de tous les foies est prélevée, déposée dans un pilulier et placée dans un four pendant un court laps de temps. Selon la quantité d'exsudats observée à l'œil nu les foies sont triés et orientés vers des valorisations différentes. L'évaluation de la fonte à la cuisson par les méthodes en verrine ou en pilulier est dépendante des conditions de chauffage: température et durée. Néanmoins, dans des conditions similaires, il existe une corrélation significative entre les deux méthodes $\left(r^{2}=0,78\right)$ (Goullieux 2007).

Le qualimètre (Salladare 1978) a été parfois utilisé par certaines entreprises, mais dans une moindre mesure que le test en « pilulier». Il s'agit d'un appareil de mesure de l'impédance électrique entre deux électrodes positionnées dans le foie gras. Dans un foie présentant une stéatose, les lipides accumulés dans le tissu hépatique augmentent la résistance au passage du courant. La taille des inclusions lipidiques au sein du cytosol joue aussi un rôle sur le niveau de résistance électrique. Aussi, la mesure de l'impédance par le qualimètre permet d'approcher à la fois le niveau et l'état d'engraissement hépatique. En partant de l'hypothèse que la richesse en lipides $\mathrm{du}$ foie comme la taille des inclusions lipidiques au sein des hépatocytes influent sur l'exsudation lipidique, cette mesure peut constituer aussi un élément de prédiction de la fonte à la cuisson. Dans des conditions d'utilisation bien standardisées (éviscération à froid), une bonne corrélation est observée entre les valeurs d'impédance obtenue au qualimètre et les résultats des tests de fonte en verrine $(\mathrm{r}=0,85)$. Une classification des foies basée sur la mesure de l'impédance a été proposée par Labie (1983). Néanmoins, Babilé et Auvergne (1986) ont montré une grande variabilité des résultats obtenus et un pourcentage élevé d'erreur. En effet, les résultats sont très dépendants de la température et ne permettent pas de trier correctement les foies gras au moment de l'éviscération. Au mieux, le qualimètre permettrait de distinguer des foies très fondants des foies très peu fondants, mais ne permettrait pas de classer les foies intermédiaires, ce qui limite fortement l'intérêt de son usage en industrie.

La Spectrométrie dans le Proche InfraRouge (SPIR) est actuellement étudiée, en conditions expérimentales, comme outil de prédiction du taux de fonte. Les premiers résultats de prédiction réalisée à l'aide de spectres acquis soit à partir d'un échantillon broyé de foie gras, soit à partir de la surface du foie, montrent de bonnes corrélations avec le taux de fonte apprécié par le test « boîte » $(\mathrm{r}=$ 0,87 sur l'échantillon broyé ; $r=0,85$ sur la surface du foie ; Marie-Etancelin, résultats non publiés). Ces corrélations sont comprises entre celles obtenues sur le même dispositif expérimental, pour la prédiction par la SPIR du taux de lipides et de protéines (Marie-Etancelin et al 2010a), et donc potentiellement exploitables.

\section{2 / Facteurs biologiques de la variation de la fonte lipi- dique à la cuisson}

\section{1 / Les facteurs d'élevage}

La préparation au gavage consiste à rationner les animaux en fin de phase d'élevage en contrôlant leur accès à la nourriture : soit celle-ci est disponible à volonté mais uniquement pendant un laps de temps réduit (on parle alors de rationnement horaire); soit elle est présente en quantité limitée (on parle alors de rationnement quantitatif). Enfin, une combinaison de ces deux types de pratiques peut également être mise en place, il s'agit du rationnement horo-quantitatif. Cette restriction conduit les animaux à augmenter leur capacité d'ingestion sur une période courte et induit une dilatation de l'œsophage, ce qui favorisera l'ingestion de grandes quantités de nourriture dès le début du gavage chez le canard (Babilé et al 1987) comme chez l'oie (Dubois et al 1996). De plus, une distribution importante d'aliment en toute fin d'élevage grâce à un rationnement quantitatif à forte progression permet une meilleure valorisation du maïs au cours du gavage tout en maintenant le taux de fonte (Robin et al 2002).

Une détérioration du rendement technologique est observée chez des animaux jeunes à l'entrée en gavage, aussi bien chez le canard mulard (Auvergne 1992, Setiawan 1994) que chez le canard de Barbarie (Auvergne 1992). Une mise en gavage précoce (à 11 vs 13 semaines d'âge) pourrait provoquer une compétition entre la fin de la croissance musculaire et le développement de la stéatose hépatique chez les animaux. En effet, la masse musculaire pectorale des palmipèdes se développe tardivement, entre 10 et 12 semaines, et le développement reste notable jusqu'à 15 semaines $(\mathrm{Au}-$ vergne 1992). L'alimentation riche en glucides et pauvre en protéines au cours du gavage induit une compétition entre les besoins protéiques des muscles pectoraux et les besoins du foie en réponse 
à la lipogenèse accrue (Babilé et al 1993, 1994). Celle-ci est d'autant plus accentuée en début de gavage lorsque l'ingéré en maïs est insuffisant pour couvrir les besoins protéiques des animaux. Dans le foie, cela se traduit par un plus faible rapport lipides/protéines qui pourrait participer à une détérioration des performances technologiques à la cuisson des foies gras de canard (Baudonnet 1993, Setiawan 1994). De plus, Robin et Castaing (1998a) ont montré que, lors d'une conduite alimentaire fortement restrictive pendant la phase d'élevage (rationnement quantitatif dès l'âge de 8 semaines), le taux de mortalité et le nombre d'animaux non valorisés sont supérieurs à ceux obtenus lors d'une conduite d'élevage intensive (rationnement horaire et non quantitatif mis en place à l'âge de 10 semaines). Après gavage, les poids des carcasses et des foies sont pénalisés de l'ordre de 7 et $10 \%$ respectivement (Robin et Castaing 1998a). Néanmoins, le taux de fonte pourrait globalement être diminué avec les poids de foies plus faibles (non évalué par Robin et Castaing 1998a).

Une ingestion importante dès le début du gavage du canard mulard (Baudonnet 1993) et du canard de Barbarie (Babilé 1989) permet un gain de poids de foie supérieur en fin de gavage et tend à améliorer la qualité technologique des foies gras. A l'inverse, une diminution de l'ingestion de maïs en fin de gavage diminue la qualité technologique des foies gras de canard mulard de 5 points, qui s'explique en partie par une différence de poids de foie (Latil et al 1996). De plus, la courbe de gavage a une influence forte sur les performances de poids de foie et sur ses qualités. Cette courbe représente les quantités de maïs ingéré à chaque repas au cours du gavage. Elle est caractérisée par la quantité ingérée au premier repas, une pente en début de gavage qui traduit l'augmentation de la quantité ingérée de maïs entre deux repas consécutifs, un palier final qui correspond à la quantité maximale ingérée. Une comparaison de cinq modèles de courbes de gavage (à quantité de maïs ingéré constante) a été réalisée par Robin et Castaing (2000). L'augmentation rapide de la ration de gavage peut entraîner des difficultés de digestion et un palier final de faible niveau de maïs ingéré, qui ont pour conséquence une diminution $\mathrm{du}$ poids de foie et du rendement technologique. En revanche, une pente initiale plus faible mais poursuivie sur une période plus longue permet d'accompagner la totalité des animaux. Les quantités ingérées élevées en fin de gavage permettent d'augmenter les poids de foie. Le modèle présentant une succession de paliers avec un dernier palier élevé permet une bonne performance en termes de poids de foie et aussi de rendement technologique (associé à une plus faible teneur en lipides) (Robin et Castaing 2000).

Une étude sur la conduite de gavage a comparé les performances technologiques des foies gras de canards mulards selon deux modalités : un gavage à 24 repas (12 jours de gavage) d'intensité forte et un gavage à 28 repas (14 jours de gavage) d'intensité faible (Setiawan 1994). Pour des poids de foie équivalents, l'augmentation de la fonte à la cuisson dans le cas du gavage moins intensif pourrait être liée au nombre plus important de repas et donc à l'allongement de la durée de gavage. Une autre hypothèse pour expliquer l'augmentation de la fonte lors d'un gavage peu intensif est que la couverture des besoins protéiques en début de gavage est également différée. Labie et al (1989) avaient par ailleurs observé par des méthodes histologiques des ruptures des membranes cellulaires après deux semaines de gavage. De la même façon, chez l'oie, un allongement de la durée de gavage (14, 18 et 21 jours) pénalise le rendement à la cuisson de 8 points (Bouillier-Oudot et al 2002), mais conduit également à des foies de poids plus important. De même, Robin et al (2002) ont montré qu'une réduction de la durée de gavage conduisait à une diminution de la fonte liée à une diminution du taux de lipides dans les foies gras.

A poids de foie équivalents, une diminution de la fonte à la cuisson est associée à une teneur inférieure de l'enzyme Lactate DésHydrogénase (LDH) sérique chez des animaux gavés pendant 14 jours par rapport à ceux gavés 20 jours (Babilé 1989). L'augmentation de la teneur en LDH sérique chez les animaux gavés sur une plus longue durée est le signe de lésions des membranes cellulaires et d'un accroissement de leur perméabilité (Auvergne 1992). Actuellement, la sélection génétique des animaux et l'amélioration des conduites d'élevage (notamment par la préparation au gavage avec rationnement horaire) permettent une forte réduction de la durée de gavage (12,5 j soit 25 repas ; RENAPALM 2012), et donc réduirait le taux de fonte.

De façon à pallier une carence en protéines lors du gavage, l'alimentation peut être supplémentée en protéines ou acides aminés. Des études ont montré que l'addition de protéines dans l'alimentation des animaux en gavage améliore le gain de poids du foie ainsi que le rendement technologique d'environ 10 points chez l'oie (Blum et al 1971, Nir et al 1973, Nitsan et al 1973). L'addition de protéines compenserait le déficit en protéines dû à l'utilisation exclusive du maïs (en particulier au début du gavage) et permettrait, selon Nir et Nitsan (1976), d'assurer une bonne intégrité des membranes des hépa- tocytes qui résisteraient alors mieux aux traitements thermiques. En revanche, l'addition de poudre de lait dans la pâtée de gavage n'améliore pas le rendement technologique (Robin et Castaing 1998b).

\section{2 / Le poids et la composition biochimique}

Chez l'oie et le canard, il existe une corrélation phénotypique positive et élevée (allant de $+0,67$ à $+0,97$ ) entre le poids de foie et le taux de fonte lipidique à la cuisson (Nir et Nitsan 1976, Barraud et Salladarre 1980, Babilé 1989, Blum et al 1990, Rousselot-Pailley et al 1993, Roussely et al 1993, Poujardieu et al 1994, Marie-Etancelin et al 2008a). Par exemple, dans un lot unique pour lequel les conditions d'élevage, de gavage et d'abattage ont été standardisées au mieux, la variabilité du poids de foie explique $33 \%$ de la variabilité de la fonte lipidique à la cuisson (figure 2). Si l'on considère ensuite une gamme de poids de foie potentiellement retenue pour la transformation en foie entier, la variabilité de la fonte à la cuisson reste élevée (coefficient de variation $=54 \%$ ), alors que le poids de foie n'explique que $14 \%$ de cette variabilité. Ainsi, plus de $80 \%$ de la variabilité de la fonte du foie gras dans la zone de poids à forte valeur ajoutée ne sont pas maîtrisés (Molette, communication personnelle).

De la même façon, sur une large gamme de poids de foie, la fonte à la cuisson est corrélée positivement avec le taux de lipides, lui-même fortement corrélé avec le poids de foie, (Rousselot-Pailley et al 1992, Théron et al 2012). Néanmoins, à poids de foie équivalents et pour une même teneur en lipides, une grande variabilité est observée dans la réponse au traitement thermique (Théron 2011).

Le rapport protéines sur lipides est relié à la qualité technologique des foies gras d'oie (Blum et al 1990). Il traduirait la capacité des structures non lipidiques à maintenir les inclusions lipidiques et ainsi à réduire la fonte à la cuisson. Une forte corrélation est trouvée entre le rendement technologique et le rapport protéines sur lipides chez le canard de Barbarie $\left(\mathrm{r}=0,78\right.$ à $55^{\circ} \mathrm{C}$ et $\mathrm{r}=0,83$ à $\left.105^{\circ} \mathrm{C}\right)$ et le canard mulard $\left(\mathrm{r}=0,91\right.$ à $55^{\circ} \mathrm{C}$ et $\mathrm{r}=$ 0,93 à $105^{\circ} \mathrm{C}$ ) (Baudonnet 1993). Chez l'oie, le taux de fonte est également corrélé négativement avec les protéines $(\mathrm{r}=$ - 0,$62 ;-0,71 ;-0,74$; pour Nir et Nitsan 1976, Blum et al 1990, Bouillier-Oudot et al 2008 respectivement). Chez le canard, une relation similaire entre taux de fonte et taux de protéines a été mise en évidence bien que plus faible $(\mathrm{r}=-0,54$; Théron $e t$ al 2012).

Une équation de prédiction du rendement technologique du foie gras de canard 
Figure 2. Relation entre le poids et la fonte lipidique du foie gras de canard pour des animaux élevés, gavés et abattus dans les mêmes conditions ( $n=150)$.

A : relation pour l'ensemble des foies. B : relation dans la gamme de poids de foie retenue pour une transformation en foies gras entiers (stérilisation ou pasteurisation) $(\mathrm{n}=70)$.

CV : Coefficient de Variation.
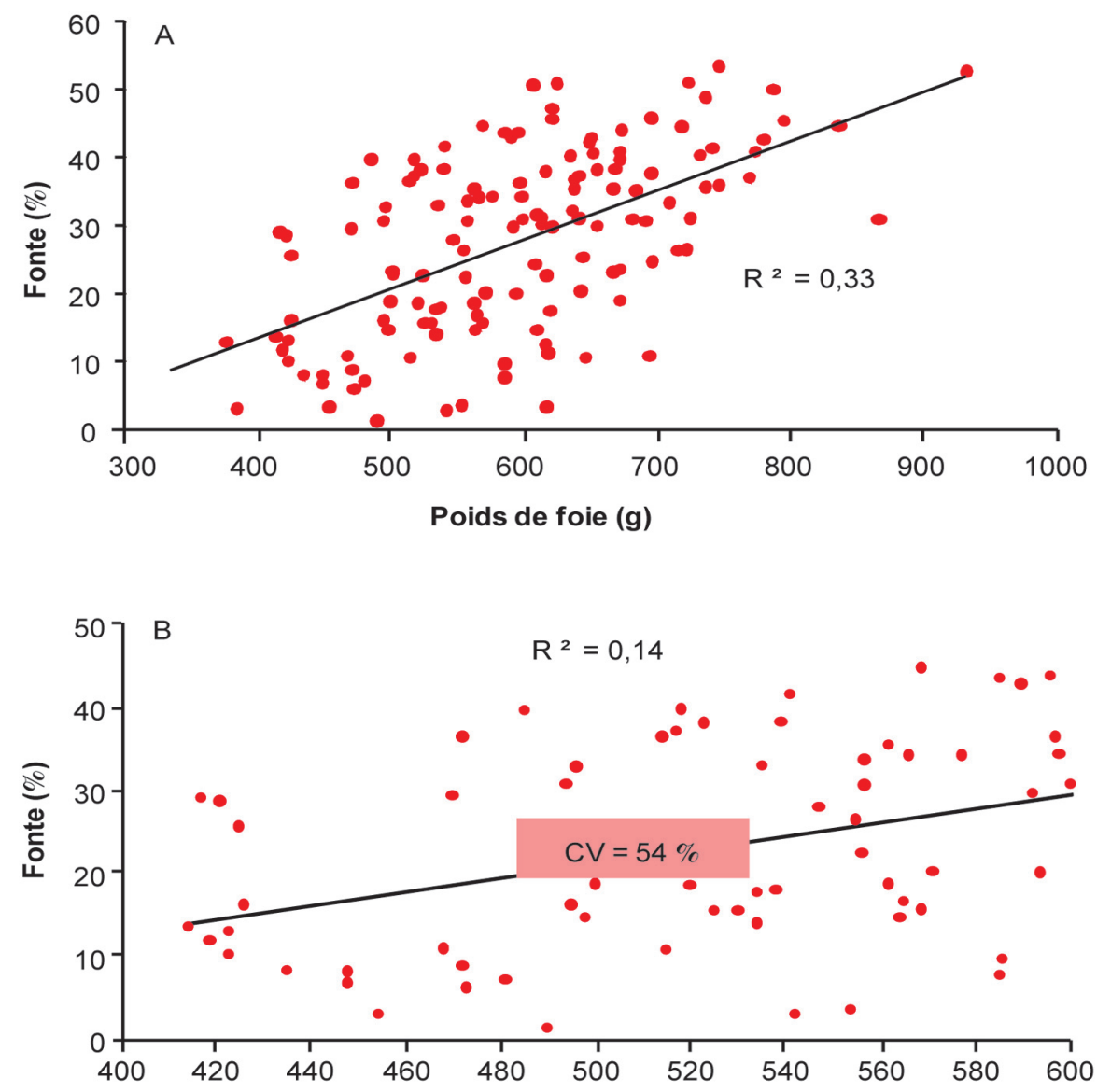

Poids de foie $(g)$

a été proposée à partir de la composition chimique des foies. Bien que de nombreuses variables soient disponibles (y compris les teneurs en lactate, glucose et les profils des lipides neutres), seuls contribuaient significativement au modèle la teneur en Matière Sèche (MS), le taux de protéines (PROT) et le Poids De Foie (PDF). Toutefois, le modèle proposé (Rendement $=-1968+61,68$ MS - 0,46 $\mathrm{MS}^{2}$ - 0,04 PDF + 3,75 PROT) ne permet d'expliquer que $43 \%$ de la variabilité du rendement technologique (Théron et al 2012).

\section{3 / Comparaison de génotypes et déterminisme génétique}

Les principales espèces de palmipèdes utilisées pour la production de foie gras sont l'oie landaise (Anser anser), le canard de Barbarie (Cairina moschata) et très majoritairement le canard mulard (Cairina moschata $x$ Anas plathyrynchos). En termes d'aptitudes au gavage et à la transformation, les foies gras issus de ces différentes espèces diffèrent principalement par le poids de foie chaud (c'est-à- doute par un taux d'engraissement plus élevé $(62,6 \%)$ des foies de canards de Barbarie. Au final, les foies gras issus de canards mulards se situent en position intermédiaire entre le canard de Barbarie et l'oie en ce qui concerne les performances de poids de foie (en moyenne $600 \mathrm{~g}$ ). Leur position également intermédiaire pour la fonte lipidique à la cuisson est à mettre en relation avec leur taux d'engraissement $(60,5 \%)$. Au sein des canards mulards, ceux issus de mères de type génétique Tsaiya (canard commun de type Asiatique) montrent de meilleures qualités technologiques, avec un taux de fonte de l'ordre de $35 \%$ mais un poids de foie gras faible (440 $\mathrm{g}$ en moyenne) que ceux issus des autres types génétiques (canards mulards issus de femelles communes Pékin ou des 2 types de femelles communes croisées, Pékin $\times$ Tsaiya ou Tsaiya $\times$ Pékin), pour lesquels les taux de fonte varient entre 38 et $46 \%$ et les poids de foies entre 560 et $640 \mathrm{~g}$ (Rouvier et al 1994). Ainsi, au sein de cet essai chez des canards mulards, on retrouve la corrélation positive entre le poids du foie et les taux de fonte puisque la correction des résultats de fonte par le poids de foie en co-variable supprime les différences significatives entre types génétiques (Setiawan 1994).

Le déterminisme génétique de la fonte lipidique à la cuisson du foie gras est résumé dans le tableau 1 . Le taux de fonte du foie de canard de Barbarie présente une forte variabilité génétique puisque les estimations d'héritabilité varient entre 0,23 (Babilé 1989) et 0,59 (Fernandez 1988). Chez le canard mulard, les estimations de paramètres génétiques sont réalisées dans les lignées parentes (soit la lignée paternelle Barbarie - Cairina moschata -, soit la lignée maternelle commune - Anas plathyrynchos dont font partie les canards Pékin et Tsaiya, soit les deux lignées) puisque la sélection a lieu dans ces lignées (MarieEtancelin et al 2008b). Ainsi, Poujardieu et al (1994) ont estimé à 0,18 l'héritabilité du taux de fonte du foie de canard mulard dans la population commune, avec une corrélation génétique élevée avec le poids de foie $(0,89)$. Lorsque les estimations d'héritabilité de performances du canard mulard sont réalisées simultanément dans les deux voies parentales, elles apparaissent très fréquemment plus faibles dans la voie paternelle (Barbarie) que dans la voie maternelle (commune) (Chapuis et Larzul 2008, Marie-Etancelin et al 2011). Ainsi, le taux de fonte du foie de canard mulard présente une héritabilité moyenne sur la voie maternelle $(0,19)$ et faible sur la voie paternelle $(0,08)$, et des corrélations génétiques fortes à très fortes avec le poids de foie $(\approx+0,80)$, le taux de lipides $(\approx+0,90)$ et le taux de protéines $(\approx-0,88)$, très proches dans les deux voies parentales. 
Tableau 1. Héritabilité $\left(h^{2}\right)$ du taux de fonte et ses corrélations génétiques $\left(r_{g}\right)$ avec les caractéristiques du foie gras, comparées chez différentes espèces de canards.

\begin{tabular}{|c|l|c|c|c|}
\hline \multirow{2}{*}{} & \multirow{2}{*}{ Caractères } & \multirow{2}{*}{ Barbarie } & \multicolumn{2}{|c|}{ Mulard } \\
\cline { 4 - 5 } & & $\begin{array}{c}\text { Lignée maternelle } \\
\text { commune }\end{array}$ & $\begin{array}{c}\text { Lignée paternelle } \\
\text { Barbarie }\end{array}$ \\
\hline \multirow{2}{*}{$\mathbf{h}^{2}$} & Taux de fonte & $0,59^{(\mathrm{a})}$ & $0,18^{(\mathrm{c})}$ & $0,08^{(\mathrm{d})}$ \\
\hline \multirow{2}{*}{$\mathbf{r}_{\mathbf{g}}$} & Poids de foie & & $0,19^{(\mathrm{d})}$ & $+0,79^{(\mathrm{d})}$ \\
\cline { 4 - 5 } & taux \\
fonte & Taux de lipides & & $+0,80^{(\mathrm{d})}$ & $+0,93^{(\mathrm{d})}$ \\
\cline { 4 - 6 } & Taux de protéines & & $+0,87^{(\mathrm{d})}$ & $-0,89^{(\mathrm{d})}$ \\
\cline { 2 - 4 } & Taux de collagène & & $-0,86^{(\mathrm{d})}$ & $-0,51^{(\mathrm{d})}$ \\
\hline
\end{tabular}

${ }^{(a)}$ Fernandez 1988, ${ }^{(b)}$ Babilé 1989, ${ }^{(c)}$ Poujardieu et al 1994, ${ }^{(\mathrm{d})}$ Marie-Etancelin et al 2011.

Si la couleur semble peu liée génétiquement au taux de fonte, le taux de collagène des foies présente une corrélation significative négative avec le taux de fonte $(\approx-0,53)$ (Marie-Etancelin et al 2011). Par ailleurs, à partir d'une combinaison linéaire du poids de foie, des taux de lipides et protéines prédits par la SPIR, la prédiction du taux de fonte est très fortement liée (plus de $+0,90$ dans les deux populations parentales) avec le taux de fonte vrai (Marie-Etancelin et al 2010b). De façon indirecte, il semble donc possible de classer génétiquement les animaux selon le taux de fonte du foie.

Plus récemment, une étude portant sur la recherche de zones chromosomiques à effet quantitatif (QTL pour « Quantitative Trait Locus ») sur la qualité technologique des foies gras a été menée chez le canard mulard (Kileh-Wais et al 2013). Ainsi, 34 QTL spécifiques des caractères d'aptitude au gavage et de qualité des produits ont pu être définis, dont 2 QTL pour le taux de fonte identifiés sur les chromosomes 2 et 9 (Kileh-Wais et al 2013) au seuil de 5\% au niveau du chromosome. Les recherches actuelles visent à identifier les gènes et les voies métaboliques sous-jacents à ces QTL, par des études de transcrits et de protéines.

\section{3 / Facteurs de variation technologiques de la fonte lipidique à la cuisson}

\section{1 / Les facteurs peri mortem}

Les facteurs peri mortem, par le stress et les modifications physiologiques qu'ils peuvent induire chez les animaux, sont susceptibles d'influencer la qualité technologique des produits finis.

Le respect d'une certaine durée de jeûne précédant l'abattage des animaux assure la vidange du tractus digestif et en conséquence, diminue le risque de contamination microbienne des carcasses lors de l'éviscération. Cette vidange est plus rapide chez l'oie (RousselotPailley et al 1992) que chez le canard de Barbarie (Auvergne et al 1993), avec respectivement des délais nécessaires d'environ 12 et 24 heures. Lors d'une augmentation du délai post-prandial avant l'abattage, une diminution du poids vif des animaux et du poids de foie est observée, associée à une moindre qualité technologique du foie gras d'oie, jusqu'à 6 points de moins (Rousselot-Pailley et al 1992), comme du foie gras de canard de Barbarie, jusqu'à 10 points de moins (Babilé 1989, Baudonnet-Lenfant et al 1991).

Les conditions d'abattage influencent la construction des qualités des produits carnés, et de nombreuses études ont été menées sur l'impact du stress lié aux conditions de transport et de déchargement. Ainsi, chez le canard mulard, une augmentation du temps de transport (150 vs 30 minutes) améliore la qualité technologique du foie gras de 6 points, sans que ce résultat ne soit relié à son poids (Fernandez et al 2011). En revanche, dans les mêmes conditions expérimentales, il a été montré que le temps d'attente sur le quai de déchargement (15 vs 120 minutes) n'a pas d'impact sur la fonte lipidique à la cuisson. Enfin, une étude de Fernandez et al (2009) a comparé l'influence de différentes méthodes d'étourdissement (dans un bain d'eau électrifiée, par pince bitemporale - « tête seulement », mécanique avec une broche perforante à ressort et en atmosphère contrôlée) sur la qualité technologique des foies gras. Il en ressort que la fonte à la cuisson du foie gras de canard ou d'oie n'est pas modifiée par la méthode d'étourdissement employée.

\section{2 / Les facteurs post mortem}

Lorsque le foie est retiré de la carcasse immédiatement en fin de chaîne d'abattage, l'éviscération est dite « à chaud ». En revanche, si la carcasse est placée en réfrigération avant le retrait du foie, on parle d'éviscération "à froid». La pratique de l'éviscération à froid détériore le rendement technologique et favorise donc la fonte lipidique à la cuisson du foie gras d'oie (Babilé et Auvergne 1986, Rousselot-Pailley et al 1992) et de canard mulard (Castaing et Robin 1990). L'hypothèse avancée par les auteurs pour expliquer la détérioration du rendement technologique dans ce cas porte sur le refroidissement plus lent du foie à l'intérieur de la carcasse, entraînant le maintien en période post mortem d'une activité importante des enzymes des catabolismes protéique, lipidique et glucidique. Il est observé notamment une teneur accrue en acides gras libres lors d'une éviscération à froid (Bouillier-Oudot et al 2002). Cela concernerait également les activités glycogénolytiques avec une diminution des réserves en glycogène au cours de la réfrigération (BouillierOudot et al 2004). Néanmoins, les mécanismes reliant la teneur en glycogène à la fonte lipidique à la cuisson ne sont pas identifiés à notre connaissance.

Une comparaison de l'impact de la valeur stérilisatrice (celle-ci permet de mesurer l'efficacité du procédé de stérilisation) sur le rendement technologique a été réalisée à partir de foies gras d'oie (Leprettre et al 2002). Les résultats montrent une diminution de la fonte à la cuisson pour des valeurs stérilisatrices plus faibles $(0,25$ vs 0,49 et 0,77$)$, tout en assurant une bonne stabilité des conserves. Plus récemment, une comparaison des effets de la pasteurisation et de la stérilisation sur le niveau et la variabilité de la fonte à la cuisson de foie gras de canard a été conduite (Molette, communication personnelle). Les résultats ont montré que la stérilisation induit une fonte à la cuisson plus élevée que la pasteurisation de 10 points environ, sans que la variabilité interindividuelle ne soit modifiée puisque la corrélation entre les valeurs de rendement obtenues aux deux températures de cuisson est très élevée $(\mathrm{r}=0,90 \mathrm{P}<0,001)$.

\section{4 / Mécanismes impliqués dans la variabilité de la fonte lipidique à la cuisson}

La recherche des mécanismes impliqués dans la variabilité de la fonte lipidique à la cuisson s'est d'abord orientée vers la composante lipidique des foies puisque les lipides représentent plus de $50 \%$ de la matière fraîche du foie gras. 
L'hypothèse d'une fragilité des membranes des hépatocytes a longtemps été évoquée pour expliquer la susceptibilité de certains foies gras à fondre à la cuisson. Cazeils et al (1999a) ont montré une augmentation du rapport cholestérol/ phospholipides des membranes des hépatocytes de l'oie au cours du gavage, en relation avec une profonde modification de leur composition en acides gras, avec notamment une forte accumulation d'acide oléique. Ces modifications concomitantes permettraient dans une certaine mesure d'assurer la stabilité du niveau de fluidité membranaire (Cooper et Strauss 1984). Cependant si le rapport cholestérol/phospholipides membranaires peut traduire des mécanismes de compensation de la fragilité des membranes induite par le gavage, il apparaît qu'une forte augmentation de ce rapport est associée à une dégradation du rendement technologique. Le rapport cholestérol/phospholipides membranaires semble ainsi être un bon indicateur de la fonte, avec une corrélation de - 0,73 entre les deux (Cazeils et al 1999b). Mais si ce résultat présente une forte significativité dans les études portant sur le foie gras d'oie, il n'a pas pu être vérifié chez le canard mulard. Enfin, l'analyse du profil en acides gras des phospholipides membranaires en relation avec le rendement technologique n'a pas permis d'établir un lien entre ces deux paramètres (Cazeils 2000).

Plus récemment, d'une part, Théron (2011) et Bonnefont et al (2013) ont étudié l'expression des protéines hépatiques et des métabolites hydrophiles précocement post mortem et, d'autre part, Théron et al (2013) ont étudié l'évolution post mortem de ces protéines au cours de la réfrigération avant transformation des foies. Pour avoir un aperçu assez large de l'implication des protéines dans le phénomène de perte à la cuisson, les auteurs ont étudié séparément deux fractions protéiques différant par leur solubilité et adapté la méthode de séparation pour chacune de ces fractions. Les profils d'expression des protéines des deux fractions protéiques et des métabolites hydrophiles ont été comparés entre des foies présentant des taux de fonte différents, de sorte à identifier des différences qui expliquent le phénomène d'exsudation lipidique pendant la cuisson.

Précocement post mortem, l'analyse protéomique a révélé quarante-sept spots exprimés de manière différentielle entre des foies à fonte élevée et des foies à fonte faible. Treize protéines ont pu être identifiées par spectrométrie de masse. D'une part, il y a une surexpression des protéines impliquées dans le métabolisme glycolytique (enolase alpha, triose phosphate isomérase 1) dans les foies à fonte faible. D'autre part, dans les foies à fonte élevée, les protéines surexprimées sont principalement impliquées dans les processus d'oxydoréduction (par exemple, peroxiredoxine 3, superoxyde dismutase-1). Les auteurs suggèrent que ces foies pourraient avoir atteint une phase de saturation en matière d'accumulation de lipides (Théron 2011). Dans la fraction non soluble, une surexpression de protéines ribosomales (treize groupes de protéines) est rapportée pour les foies à fonte faible. Au contraire, une surabondance de la protéine "heat shock $27 \gg$ (HSP27) et de l'isoforme alpha de la calponine-1 a été mise en évidence dans les foies à fonte élevée. Les résultats obtenus à partir de deux fractions protéiques avec deux technologies différentes sont concordants. En effet, dans les foies qui ont une fonte lipidique à la cuisson élevée, il semble que les protéines surexprimées soient principalement impliquées dans les mécanismes de défense afin d'avoir un effet protecteur sur l'intégrité des hépatocytes. Au contraire, dans les foies qui ont une faible fonte à la cuisson, les expressions de protéines semblent révéler des processus anaboliques. En effet, chez l'oie, ces mêmes protéines ribosomales sont surexprimées au cours du gavage (Zhu et al 2011). Les auteurs ont émis l'hypothèse que les différences dans la capacité d'accumuler des lipides dans le foie peuvent mener à divers états de développement de la stéatose après une période donnée de gavage, ce qui expliquerait une partie de la variabilité de la qualité technologique observée dans des conditions industrielles (Théron 2011). Ces résultats sont également en accord avec les observations pratiques montrant qu'une réduction de la durée de gavage améliore le rendement technologique des foies gras de canard (Bouillier-Oudot et al 2002).

Le même groupe d'auteurs a étudié les métabolites hydrophiles des hépatocytes (Bonnefont et al 2013). Ils ont montré par Résonance Magnétique du Noyau (RMN) que précocement postmortem, six métabolites avaient des concentrations supérieures dans les foies à fonte élevée (glucose, lactate, alanine, ATP, acétate et taurine ou bétaïne) et que sept métabolites avaient des concentrations supérieures dans les foies à fonte faible (choline, phosphocholine, glycérophosphocholine, myoinositol, succinate, glutamate et glycérol). Ils ont aussi montré que le glycogène était plus abondant dans les foies à fonte faible. Ils ont émis l'hypothèse que le stockage d'énergie sous forme de glycogène serait plus élevé dans les foies à fonte faible car sa concentration est supérieure, alors que l'hydrolyse du glycogène, en lactate et glucose, serait plus activée dans les foies à fonte élevée car les concentrations de ces deux métabolites sont plus faibles dans ce groupe de foies. Les teneurs plus élevées en précurseurs de phospholipides membranaires (choline, phosphocholine et glycérophosphocholine) dans les foies à fonte faible suggèrent d'après eux une meilleure intégrité des membranes plasmiques, ce qui pourrait expliquer la plus grande rétention des lipides lors de la cuisson dans ce groupe de foies.

Une diminution de la teneur en glutamate et une augmentation de la teneur en alanine a été mise en évidence dans les foies à fonte élevée. Or, chez le porc, ce profil protéique dans les muscles se traduit par un état protéolytique avancé (Lopez-Bote et al 2008). Ainsi, Bonnefont et al (2013) émettent l'hypothèse que les foies à fonte élevée seraient plus enclins à la dégradation protéique que les foies à fonte faible. Il semblerait que leurs observations réalisées précocement post mortem se maintiennent après le refroidissement des foies. Ainsi les mesures précoces des métabolites hydrophiles des foies gras permettent d'expliquer une partie de la variabilité des pertes lipidiques à la cuisson.

Théron et al (2013) ont étudié pour la première fois l'évolution du protéome du foie gras post mortem. L'expression globale de la fraction soluble à faible force ionique montre une diminution après refroidissement (moindre expression des protéines impliquées dans les processus d'oxydo-réduction, des protéines à activités protéolytiques et des protéines chaperones après 6 heures de réfrigération). Ces résultats peuvent être rapprochés des observations réalisées dans le muscle squelettique et qui montrent que l'évolution post mortem se traduit par une diminution du système de défense antioxydant chez le bœuf (Renerre et al 1996) et la dinde (Renerre et al 1999). Par ailleurs, dans la fraction non soluble à faible force ionique, Théron et al (2013) ont mis en évidence une forte représentativité de protéines du cytosquelette à 6 heures post mortem dans les foies qui ont une fonte élevée. Les auteurs émettent l'hypothèse que cette surabondance de protéines cytosquelettiques serait le résultat de l'hydrolyse de ces protéines avec l'apparition de fragments. L'hydrolyse des protéines du cytosquelette est impliquée dans le déterminisme de la capacité de rétention en eau de la viande de porc (Kristensen et Purslow 2001), elle peut avoir lieu dès 45 minutes et jusqu'à 6 heures post mortem (Melody et al 2004). Ces résultats impliqueraient des mécanismes de protéolyse dans les mécanismes de la fonte lipidique à la cuisson du foie gras et confirmeraient ainsi ceux obtenus dans la fraction protéique soluble à faible force ionique. L'ensemble de ces résultats est cohérent avec des observations pra- 
tiques qui suggèrent qu'une réfrigération plus rapide des foies gras (par la mise en œuvre de l'éviscération à chaud en comparaison de l'éviscération à froid) a un effet positif sur le rendement technologique (Bouillier-Oudot et al 2002). Dans ce cas, la réduction de la fonte lipidique à la cuisson pourrait s'expliquer par une stabilisation plus rapide du tissu et notamment par une inhibition des réactions protéolytiques dans le tissu. A l'inverse, la réfrigération ne semble pas suffisante pour enrayer des mécanismes sans doute déjà enclenchés précocement dans les foies gras qui ont une fonte élevée (Rousselot-Pailley et al 1992). La conséquence d'une moindre efficacité du refroidissement sur ces foies gras serait une protéolyse plus intense, se traduisant par la lyse du cytosquelette. Le tissu fragilisé serait alors plus sensible aux contraintes mécaniques de la cuisson conduisant à une augmentation de la fonte à la cuisson.

\section{Conclusion}

L'étude bibliographique sur la fonte lipidique à la cuisson du foie gras révèle que de nombreuses études ont été menées sur le sujet et ont permis de définir des facteurs de variation, notamment zootechniques et technologiques (figure 3 ). Ces travaux ont permis de modifier certaines pratiques comme, par exemple, l'éviscération à froid qui avait un impact négatif sur le rendement technologique que ce soit pour le foie gras de canard ou d'oie. Pourtant, lorsque ces facteurs sont maîtrisés, il demeure une variabilité importante et non contrôlée du rendement technologique des foies gras.

Figure 3. Synthèse des facteurs de variation du rendement technologique à partir des données de la littérature.

\section{Facteurs biologiques}

\begin{tabular}{lrr|}
\hline Génétique & Elevage & Gavage \\
Rendement : & Rendement $\pi \mathrm{si}$ & Rendement $\pi \mathrm{si}$ \\
Mulard $>$ Barbarie & - Animaux jeunes $(10$ semaines $)$ & - Durée courte (10 jours) \\
$<\mathrm{h}^{2}<0.22$ & - Teneur en lysine $\approx 7 \mathrm{~g} / \mathrm{kg}$ & - Intensité gavage $\lambda$ ou $\rightarrow$
\end{tabular}

$0,09<h^{2}<0.22$ - Teneur en lysine $\approx 7 \mathrm{~g} / \mathrm{kg}$

Rendement $\pi$ si

-Poids de foie $y$

-Taux de lipides $\searrow$

-Taux de protéines $\pi$

FACTEURS DE VARIATION

CONNUS DU RENDEMENT TECHNOLOGIQUE

Rendement $\pi$ si

- Délai post-prandial $\$

-Temps de transport $\pi$

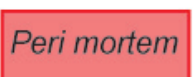

Rendement $\pi$ si

- Eviscération à chaud

-Pasteurisation (vs stérilisation)

\section{Facteurs technologiques}

Les études conduites sur le déterminisme de la variabilité de la fonte lipidique à la cuisson du foie gras de canard ont permis, en combinant différents niveaux d'approches, d'élaborer un scénario cohérent et pertinent. Les travaux se poursuivent actuellement afin de mieux connaître le rôle des protéases hépatiques dans les mécanismes de la fonte. Enfin, la recherche de gènes impliqués dans ces mécanismes est en cours que ce soit pour comprendre la régulation du métabolisme hépatique ou pour identifier des régions du génome responsables de la variabilité observée.

\section{Références}

Auvergne A., 1992. Facteurs de variation de la composition corporelle et tissulaire des canards avant et après gavage. Thèse de Doctorat d'Etat, Institut National Polytechnique de Toulouse, France, 252p.

Auvergne A., Babilé R., Remond D., Latil G., 1993. Digestibilité du maïs en gavage. Eléments de prediction du gain de poids. $1^{\text {ères }}$ Journ. Rech. Palmipèdes à Foie Gras, Bordeaux, France, 75-89.

Babilé R., 1989. La production de foies gras de canards de Barbarie (Caïrina moschata) : Aspects génétiques, nutritionnels et technologiques. Thèse de Doctorat, INP Toulouse, France, 231p.

Babilé R., Auvergne A., 1986. Qualité des foies gras : Comparaison de différentes méthodes d'appréciation de la fonte lipidique. Evolution en fonction du temps. Rec. Med. Vet., 162, 151-156.

Babilé R., Auvergne A., Delpech P., Meirieu O., 1987. Evolution de la consomma- tion de maïs au cours du gavage, incidence sur la production de foie gras de canard de Barbarie. Ann. Zoot., 36, 73-74.

Babilé R., Auvergne A., Setiawan I., Joly E., Latil G., 1993. Conduite alimentaire au cours du gavage des canards et qualité technologique $\mathrm{du}$ foie gras. $1^{\text {eres }}$ Journ. Rech. Palmipèdes à Foie Gras, Bordeaux, France, 107-116.

Babilé R., Auvergne A., Borgida L.P., Chambart G., 1994. L'apport protéique et minéral au cours du gavage est bénéfique. L'aviculteur, 81-82.

Baéza E., Marie-Etancelin C., Davail S., Diot C., 2013. La stéatose hépatique chez les palmipèdes. In : Palmipèdes à foie gras. Fortun-Lamothe L. (Ed). Dossier, INRA Prod. Anim., 26, 403-414.

Barraud C., Salladarre P., 1980. La qualité du foie gras. R.T.V.A., 160, 47-53.

Baudonnet C., 1993. Facteurs de variation de la composition biochimique et de la qualité technologique des foies gras de canards. Thèse de Doctorat d'Etat, Institut National Polytechnique de Toulouse, 182p.

Baudonnet-Lenfant C., Auvergne A., Babilé R., 1991. Influence de la durée de jeûne avant l'abattage et de poids de la mise en gavage des canards de Barbarie sur la composition chimique hépatique. Ann. Zoot., 40, 161-170.

Blum J.C., Monachon G., Leclercq B., 1971. Liver steatosis of force-fed geese as influenced by the protein level of the diet. Acta Vet. Academiae Scientarium Hungaricae, 21, 307-311.

Blum J.C., Labie C., Raynaud P., 1990. Influence du poids et de la composition chimique $\mathrm{du}$ foie gras d'oie sur la fonte mesurée après stérilisation à $104^{\circ} \mathrm{C}$. Sci. Alim., 10, 543-554.

Blum J.C., Salichon M.R., Guy G., RousselotPailley D., 1992. Comparative development, chemical composition and quality of ducks and goose 'foie gras' obtained by cramming. In: XIX World's Poultry Congress. WPSA, Amsterdam, Netherlands, 240-244. 
Bonnefont C.M.D., Guerra A., Théron L., Molette C., Canlet C., Fernandez X., 2013. Metabolomic study of fatty livers in duck: identification by ${ }^{1} \mathrm{H}-\mathrm{NMR}$ of metabolic markers associated with technological quality. Poult. Sci., submitted.

Bouillier-Oudot M., Leprettre S., Dubois J.P. Babilé R., 2002. Itinéraires post mortem et caractéristiques technologiques et organoleptiques des foies gras d'oies. $5^{\text {èmes }}$ Journ. Rech. Palmipèdes à Foie Gras, Pau, France, 172-175.

Bouillier-Oudot M., Leprettre S., Dubois J.P., Babilé R., 2004. Evolution de la composition hépatique lors du refroidissement post mortem de foies gras d'oies dans la carcasse. $6^{\text {èmes }}$ Journ. Rech. Palmipèdes à Foie Gras, Arcachon, France, 203-206.

Bouillier-Oudot M., Dubois J.P., Auvergne A., Babilé R., Fernandez X., 2008. Incidence du rythme de gavage et du sexe sur les performances zootechniques d'oies et les caractéristiques chimiques, histologiques et technologiques des foies gras. $8^{\text {èmes }}$ Journ. Rech. Palmipèdes à Foie Gras, Arcachon, France. 131-134.

Castaing J., Robin N., 1990. Alimentation énergétique du canard mulard. Rev. Alim. Anim., 440, 22-25.

Cazeils J.L., 2000. Caractérisation de la composition lipidique des membranes plasmiques des hépatocytes des foies d'oies : relation avec le rendement technologique des foies gras. Thèse de Doctorat de 1'Institut National Polytechnique de Toulouse, France, 192p.

Cazeils J.L., Bouillier-Oudot M., Auvergne A., Babilé R., 1999a. Influence de la composition biochimique de la membrane plasmique des hépatocytes sur le rendement après stérilisation des foies gras d'oies. Rev. Méd. Vét., 150, 651-654.

Cazeils J.L., Bouillier-Oudot M., Auvergne A., Candau M., Babilé R., 1999b. Lipid composition of hepatocyte plasma membranes from geese overfed with corn. Lipids, 34, 937-942.

Chapuis H., Larzul C., 2008. Comment estimer simultanément les paramètres génétiques des caractères de gavage dans les 2 lignées parentales du mulard en vue d'une sélection plus efficace ? $8^{\text {èmes }}$ Journ. Rech. Palmipèdes à Foie Gras, Arcachon, France, 29-32.

Comité Interprofessionnel des Palmipèdes à Foies Gras (CIFOG), 2011. Assemblée générale du 24 juin 2011. Rapport économique de l'année 2010,75 p

Cooper R.A., Strauss J.F., 1984. Regulation of cell membrane cholesterol. In: Physiology of membrane fluidity. Shinitzky M. (Ed), CRC Press, Boca Raton, USA, 1, 73-97.

Dubois J.P., Auvergne A., Babilé R., Verdier M., Leprettre S., Lavigne F., Vieillecroze D. 1996. Le point sur les facteurs d'amélioration de la production d'oies gavées. $2^{\text {èmes }}$ Journ. Rech. Palmipèdes à Foie Gras, Bordeaux, France, 89-92.

Fernandez X., 1988. Etude de la variabilité génétique d'une population de canards de Barbarie mâles sur les caractères de croissance et de gavage de ses descendants. Mémoire de fin d'étude, Ecole Nationale Supérieure Agronomique de Toulouse, France.

Fernandez X., Lahirigoyen E., BouillierOudot M., Vitezica Z., Auvergne A., 2009. The effects of stunning methods on product qualities in force-fed ducks and geese. 2. Fatty liver quality. Animal., 4, 139-146.
Fernandez X., Bouillier-Oudot M., Molette C., Bernadet M.D., Manse H., 2011. Duration of transport and holding in lairage at constant postprandial delay to slaughter - Effects on fatty liver and breast muscle quality in mule ducks. Poult. Sci., 90, 2360-2369.

Goullieux I., 2007. Recensement et évaluation des méthodes de mesure du taux de fonte et du rendement. Viandes Prod. Carnés, 26, 147-151.

Guy G., Rousselot-Pailley D., Gourichon D., 1995. Comparaison des performances de l'oie, du canard mulard et du canard de Barbarie soumis au gavage. Ann. Zootech., 44, 297-305.

Guy G., Hermier D., Davail S., Bely M. André J.M., Hoo-Paris R., 1999. Meat production and force-feeding ability of different types of ducks. In: $1^{\text {st }}$ World Waterfowl Conference, 1-4/12/99, Taichung, Taiwan, 462-468.

Kileh-Wais M., Elsen J.M., Vignal A., Feves K., Vignoles F., Fernandez X., Manse H., Davail S., André J.M., Bastianelli D., Bonnal L. Filangi O., Baéza E., Guéméné D., Genêt C., Bernadet M.D., Dubos F., Marie-Etancelin C., 2013. Detection of QTL controlling metabolism, meat quality, and liver quality traits of the overfed interspecific hybrid mule duck. J. Anim. Sci., 91, 588-604.

Kristensen L., Purslow P.P., 2001. The effect of ageing on the water-holding capacity of pork: role of cytoskeletal proteins. Meat Sci., $58,17-23$

Labie C., 1983. Qualité du foie gras. In : Du foie gras au cassoulet. Maîtrise et gestion de la filière palmipèdes gras. A.P.R.I.A., Paris, Centre Technique de la Conservation des Produits Agricoles, Bordeaux, France, 133-150.

Labie C., Bénard G., Gauclere B., 1989. Evolution de la stéatose hépatique chez le canard mulard au cours du gavage. Rev. Med. Vet., 140, 809.

Latil G., Auvergne A., Babilé R., 1996. Consommation du canard mulard en gavage. Relations avec les performances zootechniques et la qualité technologique du foie gras. $2^{\text {em }}$ Journ. Rech. Palmipèdes à Foie Gras, Bordeaux, France, 93-97.

Leprettre S., Dubois J.P., Bouillier-Oudot M., Babilé R., 2002. Technique de gavage des oies : Incidence de la fréquence des repas et de la durée du gavage sur les performances et les caractéristiques technologiques et organoleptiques des foies gras. $5^{\text {emes }}$ Journ. Rech. Palmipèdes à Foies Gras, Pau, France.

Lopez-Bote C.J., Toldrá F., Daza A., Ferrer J.M., Menoyo D., Silió L., Rodríguez M.C., 2008. Effect of exercise on skeletal muscle proteolytic enzyme activity and meat quality characteristics in Iberian pigs. Meat Sci., 79, 71-76.

Marie-Etancelin C., André J.M., Baéza E., Basso B., Bastianelli D., Bernadet M.D. Brun J.M., Davail S., Dubos F., Fernandez X Gontier K., Guemene D., Guy G., Manse H., Mialon M.M., Larzul C., 2008a. Dispositif de détection de QTL chez le canard commun. $8^{\text {èmes }}$ Journ. Rech. Palmipèdes à Foie Gras, Arcachon, France, 17-20.

Marie-Etancelin C., Chapuis H., Brun J.M., Larzul C., Richard M.M., Rouvier R., 2008b. Genetics and selection of mule ducks in France: A review. World Poult. Sci. J., 64, 187-207.

Marie-Etancelin C., Fernandez X., Baéza E. Bonnal L., Manse H., Chartrin P., Bernadet
M.D. Bastianelli D., 2010a Déterminisme génétique de la composition du foie gras et des magrets obtenue par spectrophotométrie dans le proche infrarouge. $9^{\text {emes }}$ Journ. Rech. Palmipèdes à Foie Gras, Bordeaux, France, 105 108.

Marie-Etancelin C., Fernandez X., Baéza E., Bonnal L., Bernadet M.D., Manse H., Chartrin P., Bastianelli D., 2010b. Genetic parameters of fatty liver and breast muscle composition predicted by near-infrared spectroscopy. $9^{\text {th }}$ World Cong. Genet. Appl. Livest. Prod., Leipzig, Allemagne, 1-6 Aout 2010, ID 779, $4 \mathrm{p}$.

Marie-Etancelin C., Basso B., Davail S., Gontier K., Fernandez X., Vitezica Z.G., Bastianelli D., Baéza E., Bernadet M.D., Guy G., Brun J.M., Legarra A., 2011. Genetic parameters of product quality and hepatic metabolism in fattened mule ducks. J. Anim. Sci., 89, 669-679.

Melody J.L., Lonergan S.M., Rowe L.J., Huiatt T.W., Mayes M.S., Huff-Lonergan E., 2004. Early postmortem biochemical factors influence tenderness and water-holding capacity of three porcine muscles. J. Anim. Sci., 82, 1195-1205.

Nir I., Nitsan Z., Vax A., 1973. The influence of force-feeding and of protein supplementation to the diet on the metabolisable energy of diets, digestibility of nutrients, nitrogen retention and digestive enzymes output in geese. Ann. Biol. Anim. Bioch. Biophys., 13, 465-479.

Nir I., Nitsan Z., 1976. Goose fatty liver composition as related to degree of steatosis, nutritional and technological treatments, and simplified method for quality estimation. Ann. Zoot., 25, 461-470.

Nitsan Z., Nir I., Dror Y., Bruckental I., 1973. The effect of forced feeding and of dietary protein level on enzymes associated with digestion, protein and carbohydrate metabolism in geese. Poult. Sci., 52, 474-481.

Poujardieu B., Guichard F., Laventure P. 1994. Paramètres génétiques de croissance et de gavage de la cane commune. Genet. Sel. Evol., 26, 468-472.

RENAPALM, 2012. Gestion technico-économique des éleveurs et gaveurs de palmipèdes à foie gras. Résultats 2012 - Campagne 2011, Rapport ITAVI, 111p.

Renerre M., Dumont F., Gatellier P., 1996. Antioxidant enzyme activities in beef in relation to oxidation of lipid and myoglobin. Meat Sci., $43,111-121$.

Renerre M., Poncet K., Mercier Y., Gatellier P., Metro B., 1999. Influence of dietary fat and vitamin $\mathrm{E}$ on antioxidant status of muscles of turkey. J. Agric. Food Chem., 47, 237-244.

Robin N., Castaing J., 1998a. Effet d'une restriction alimentaire sur les performances des canards mulards. $3^{\text {èmes }}$ Journ. Rech. Palmipèdes à Foie Gras, Pau, France, 91-94.

Robin N., Castaing J., 1998b. Incidence d'une supplémentation énergétique et proteique du maïs broyé sur la production de foie gras de canard mulard en gavage. $3^{\text {èmes }}$ Journ. Rech. Palmipèdes à Foie Gras, Pau, France, 9598.

Robin N., Castaing J., 2000. Comparaison de différents modèles de distribution du maïs broyé pour le gavage des canards mulards. $4^{\text {èmes }}$ Journ. Rech. Palmipèdes à Foie Gras, Pau, France, 121-125. 
Robin N., Peyhorgue A., Castaing J., 2002. Comparaison de différents itinéraires techniques élevage/gavage pour un gavage cours des canards mulards. $5^{\text {emes }}$ Journ Rech. Palmipèdes à Foie Gras, Pau, France, 148-151.

Rousselot-Pailley D., Guy G., Gourichon D., Sellier N., Blum J.C., 1992. Influence des conditions d'abattage et de réfrigération sur la qualité des foies gras d'oie. INRA Prod. Anim., $5,167-172$.

Rousselot-Pailley D., Rouvier R., Bartel G., 1993. Méthodologie du test de fonte des foies gras. $1^{\text {reres }}$ Journ. Rech. Palmipèdes à Foie Gras, Bordeaux, France, 75-85.

Roussely M., Guichard F., Sazy E., 1993. Performances zootechniques et qualité des produits du canard mulard: Analyse de la variabilité individuelle. $1^{\text {ires }}$ Journ. Rech. Palmipèdes à Foie Gras, Bordeaux, France, 89-105.
Rouvier R., Guy G., Rousselot-Paillet D., Poujardieu B., 1994. Genetic parameters from factorial cross breeding in two duck strains (anas platyrhynchos) Brown Tsaiya and Pekin, for growth and fatty liver traits. Br. Poult. Sci., $35,509-17$.

Salladare P., 1978. Objectivation des qualités technologiques des foies gras par mesures d'impédance bioélectrique. Thèse de Doctorat, Université Claude-Bernard, Lyon, France, $141 \mathrm{p}$.

Setiawan A., 1994. Etudes comparatives de différents types génétiques de canards mulards : croissance, composition corporelle et tissulaire, et qualité des produits obtenus avant et après gavage. Thèse de Doctorat en Sciences Qualité des Aliments, Institut National Polytechnique de Toulouse, France, 274p.

Théron L., 2011. Déterminisme biologique de la variabilité de la fonte lipidique à la cuis- son chez le canard. Thèse de Doctorat, Institut National Polytechnique de Toulouse, France, 170p.

Théron L., Cullere M., Bouillier-Oudot M., Manse H., Molette C., Fernandez X., Vitezica Z.G., 2012. Modeling the relationships between quality and biochemical composition of fatty liver in mule ducks. J. Anim. Sci., 90, 3312-3317.

Théron L., Fernandez X., Marty-Gasset N., Chambon C., Viala D., Pichereaux C., Rossignol M., Astruc T., Molette C., 2013. Proteomic analysis of duck fatty liver during post mortem storage related to the variability of fat loss during cooking of 'foie gras'. J. Agric. Food Chem., 61, 920-930.

Zhu L., Meng H., Duan X.J., Xu G.Q., Zhang J., Gong D.Q., 2011. Gene expression profile in the liver tissue of geese after overfeeding. Poult. Sci., 90, 107-117.

\title{
Résumé
}

La capacité à retenir les lipides lors de la cuisson constitue la principale qualité technologique recherchée du foie gras. La fonte se traduit par une exsudation de nature essentiellement lipidique qui a lieu lors des traitements thermiques de conservation. Différentes méthodes d'évaluation de ce rendement sont disponibles et de manière générale, les corrélations entre ces dernières sont significatives. La variabilité du rendement technologique est élevée. Des facteurs de variation identifiés sont des paramètres liés à l'élevage, au gavage ou encore aux conditions entourant l'abattage des animaux. Des études ont également permis de mettre en évidence un lien entre le rendement technologique et de nombreux paramètres : le type et les caractéristiques génétiques de l'animal, le poids du foie et sa composition chimique. Enfin, la recherche du déterminisme biologique de la variabilité de la fonte à la cuisson a conduit à proposer un mécanisme en lien avec le niveau de stéatose hépatique au moment de l'abattage.

\begin{abstract}
Melting rate after cooking of fatty liver in waterfowl

The ability to retain fat during cooking is the main technological quality of "foie gras". Melting rate results in exudation, essentially of lipidic nature which takes place during the heat treatments of conservation. Different methods that assess the melting rate are available and generally the correlations between them are significant. Variability of technological yield is high. The variation factors identified are the animal and its genetic type parameters related to the breeding period, over-feeding period or the conditions surrounding the slaughter of animals. Studies have also highlighted a link between technological performance and parameters related to the animal (genetic line and genetic characteristics of animals) and to the liver (liver weight and chemical composition). Finally, research on the biological determinism of the variability of melting rate has led to propoing a mechanism linked to the level of hepatic steatosis at the time of slaughter.
\end{abstract}

THÉRON L., BOUILLIER-OUDOT M., MARIE-ETANCELIN C., BONNEFONT C., FERNANDEZ X., MOLETTE C., 2013. La fonte lipidique du foie gras à la cuisson. In : Palmipèdes à foie gras. Fortun-Lamothe L. (Ed). Dossier, INRA Prod. Anim., 26, 415-424. 